\title{
Carnets
}

Revue électronique d'études françaises de l'APEF

Deuxième série - 5 | 2015

Imaginaires de guerre et autres conflits

\section{Andreï Makine, témoin intemporel de la guerre en Russie soviétique}

Alexia Gassin

\section{(2) OpenEdition}

Journals

Édition électronique

URL : http://journals.openedition.org/carnets/416

DOI : 10.4000/carnets.416

ISSN : 1646-7698

\section{Éditeur}

APEF

\section{Référence électronique}

Alexia Gassin, «Andreï Makine, témoin intemporel de la guerre en Russie soviétique », Carnets [En ligne], Deuxième série - 5 | 2015, mis en ligne le 30 novembre 2015, consulté le 01 mai 2019. URL: http://journals.openedition.org/carnets/416 ; DOI : 10.4000/carnets.416

Ce document a été généré automatiquement le 1 mai 2019.

\section{(c) (i) (8)}

Carnets est mis à disposition selon les termes de la licence Creative Commons - Atribution - Pas d'utilisation commerciale 4.0 International. 


\title{
Andreï Makine, témoin intemporel de la guerre en Russie soviétique
}

\author{
Alexia Gassin
}

Bien que le thème de la guerre ait été peu étudié par les spécialistes de Makine, force est de constater qu'il est omniprésent dans l'œuvre de l'écrivain, notamment dans les romans Confession d'un porte-drapeau déchu (1992), Le Testament français (1995), Requiem pour l'Est (2000) et La Vie d'un homme inconnu (2009) où l'auteur évoque successivement la Première et la Seconde Guerre mondiale, la guerre civile russe (1917-1923) et les divers conflits issus de la Guerre froide attestant des affrontements indirects entre les États-Unis et l'Union soviétique. En cela, Makine propose un large panorama des nombreuses luttes ayant eu lieu au xx $x^{\mathrm{e}}$ siècle, aussi bien en France qu'en Russie bolchévique et en U.R.S.S. Néanmoins, même si Makine se réfère plusieurs fois aux victimes françaises de la guerre 1914-1918, qu'il qualifie d'« enfants morts pour la France » (Makine, 2006 : 16) ou « tombé [s] au champ d'honneur » (Makine, 1998: 78), nous concentrerons nos propos sur les affrontements vécus par les Russes ou les Soviétiques dont Makine dépeint la dure réalité ${ }^{1}$ . Comme le souligne Geneviève Lubrez, la guerre décrite par l'auteur

entraîn[e] tout et tous dans la tourmente; riches et pauvres, soldats et paysans, hommes et femmes. Elle est le destin de tout un peuple; elle est l'aune à laquelle chacun va mesurer sa part d'être; elle est en quelque sorte le sol qui révélera à chacun sa propre vérité et à nous lecteurs la complexité de l'être ; à l'esprit en proie au doute, à l'âme blessée et souffrante elle apportera la lumière de l'essentiel qui seul donne la paix intérieure (Lubrez, $2005: 33$ ).

De ce fait, selon Murielle Lucie Clément, "Makine peut être considéré, à un certain niveau, comme un archiviste de l'histoire » (Clément, 2010 : 98), également soucieux du devoir de mémoire à l'égard des martyrs de guerre. Elle précise néanmoins que son travail n'est pas celui d'un historien. En effet,

en tant qu'écrivain, Makine n'est pas tenu de se confiner aux seuls faits historiques avérés. Ses données n'ont pas à être " objectives » comme devraient l'être celles d'un historien. Il n'a pas non plus à se mettre en cause dans son processus scriptural comme un observateur scientifique dans le face à face avec son objet d'études. Il peut cependant s'appuyer sur des points référentiels pour stimuler l'identification du lecteur (Clément, $2010: 100$ ). 
Par conséquent, alors que "Makine (...) écrit sur la guerre qu'il n'a pas connue » (Clément, 2011), il propose la « fictionnalisation d'une idée » (Clément, 2011), « basée sur une connaissance, sur un témoignage » (Clément, 2011). Dès lors, la question qui se pose est de savoir comment l'écrivain nourrit son imaginaire de guerre, reposant sur des descriptions engagées et critiques et pouvant s'inspirer en partie des archives conservées dans les conservatoires russes, à l'exemple du musée du siège de Léningrad ou musée du Blocus, évoqué dans le roman La Vie d'un homme inconnu, où

les salles (...) contenaient un fouillis d'éclats tragiques du passé, de ces années dont il était si difficile de parler. Photos, objets personnels, lettres, cahiers où des enfants qui mouraient de froid dessinaient l'herbe, les nuages d'été... Et le carnet de cet enfant-là qui avait noté la date de la mort de chacun de ses proches (Makine, 2009 : 201).

Il se peut également que Makine ait retranscrit des récits authentiques, relatant l'expérience individuelle de personnes ayant connu la guerre et ses conséquences, telle cette femme apparaissant dans Le Testament français et dans Requiem pour l'Est sous le nom de Charlotte ou de Sacha et qui, outre leur rôle de témoin d'une époque conflictuelle, serait aussi l'incarnation de la prétendue grand-mère française grâce à qui « Makine devient bilingue et découvre la culture française » (Nazarova, $2008: 13$ ).

Ainsi, même si l'auteur n'a pas observé la guerre de manière directe, son travail de chroniqueur ne se rapproche pas moins de celui des écrivains Viktor Nekrassov, officier dans un bataillon de sapeurs, et de Vassili Grossman, correspondant de guerre pour le journal Krasnaïa Zvezda (Étoile rouge). À l'instar de ces deux témoins de la Seconde Guerre mondiale et de la bataille de Stalingrad, dont les écrits s'opposent à la propagande contenue dans les manuels d'histoires, les mémoires des généraux et les discours officiels, Makine, qui se transforme en véritable journaliste d'investigation ${ }^{2}$, donne la parole à l'humain en délaissant la grande Histoire et ses mensonges au profit de l'histoire individuelle de «ces 'femmes inconnues' et ces 'hommes inconnus' (...) dont la parole est restée muette » (Makine, 2009 : 292).

6 Pour ce faire, l'auteur propose un regard extérieur sur la guerre à l'aide d'un ou de plusieurs narrateurs, externes ou internes, qui, la plupart du temps, n'ont pas directement vécu la guerre en tant que soldat. Ainsi, dans Le Testament français, Aliocha entend parler de la guerre par les membres de sa famille, en particulier par sa grandmère qui, elle-même, fut infirmière sur le front, d'abord pour la Croix-Rouge, et n'a vu la guerre qu' " en passant ", c'est-à-dire lors de son voyage de France en Russie, effectué à pied ou en train. C'est de cette façon qu'elle découvre les conséquences de la guerre civile entre les Russes blancs et les Russes rouges, sous forme de photographies vivantes, d'images gravées à tout jamais dans sa mémoire :

Ces paysans en colère qui, avec de longues perches, repoussaient une barge d'où montait une interminable plainte. On voyait des silhouettes qui, de son bord, tendaient leurs mains décharnées en direction de la berge. C'étaient les malades du typhus, abandonnés, et qui dérivaient sur leur cimetière flottant depuis plusieurs jours. À chaque tentative d'accoster, les riverains se mobilisaient pour les en empêcher. La barge reprenait sa navigation funèbre, les gens mouraient, à présent aussi de faim. Bientôt, ils n'auraient plus la force de tenter une escale, et les derniers survivants, réveillés un jour par le bruit puissant et régulier des vagues, verraient l'horizon indifférent de la Caspienne... (Makine, 1998 : 84).

7 Ces mêmes instantanés réapparaissent dans Requiem pour l'Est où le narrateur, dont le nom nous reste inconnu jusqu'à la fin du livre, est un médecin de guerre pendant la 
Guerre froide, puis un espion pour les services secrets soviétiques, qui vit la guerre à l'intérieur de bâtiments qui semblent délimiter la frontière entre son quotidien et la réalité.

Ensuite, dans Confession d'un porte-parole déchu, Kim écoute les histoires de guerre racontées par son père ou par son entourage tandis que son père, alors tireur d'élite, ne voit la guerre qu' « à travers la transparence tamisée de la lunette » (Makine, $1996: 33$ ) de son fusil, à la manière d'un cadreur qui dirigerait une caméra lors de prises de vue pour un film de guerre, présentant l'action qui se déroule sous ses yeux :

Le jeune officier range ses feuilles dans une serviette, disparaît, surgit sur le perron, le dévale allègrement, se dirige vers une moto qui l'attend dans la cour. Le soldat sursaute sur son siège en mettant le moteur en marche. L'officier se cale dans le side-car et, au même moment, comme pris d'une rêverie profonde, laisse tomber son menton sur sa poitrine. Dans la pétarade du moteur, le soldat n'a rien remarqué.

La douille vide saute, la nouvelle cartouche glisse à sa place. Le rond silencieux découpé dans la journée calme de l'été s'approche de nouveau de l'isba.

Sur le perron apparaissent les deux interlocuteurs. L'un d'eux sort un porte-cigares, l'autre fouille dans sa poche. Oui, c'est bien cela, le briquet est resté à l'intérieur. Il va le chercher... Maintenant, l'essentiel est de ne pas s'endormir !

L'officier qui vient d'ouvrir le porte-cigares le rejette soudain avec un air de dégoût et, s'accrochant à la rampe, s'affale. Son compagnon qui sort, en jouant avec le briquet, a le temps de voir les cigarettes éparpillées et, la tête renversée, s'écroule dans l'entrée.

À présent, chaque seconde compte. Mettre une gaine sur la lunette, ramasser les trois douilles et, alternant les courses brèves et les attentes figées, parvenir au taillis le plus proche (Makine, 1996 : 31-32).

Enfin, dans La Vie d'un homme inconnu, c'est Georgui Volski qui relate son expérience de la guerre au premier narrateur, Ivan Choutov, et explique comment il a vécu les premiers mois de la guerre en tant que chanteur avant d'être envoyé sur le front :

La plaine qui paraissait nue frémit et se couvrit de points. La nuit, prise au dépourvu, resta silencieuse pendant quelques secondes, puis d'un coup, éclata en fusillades. La clameur sourde d'un « hourra!" se dilata dans l'air. Le «capitaine » agita les bras, la musique résonna. Les chanteurs effacèrent par la puissance de leurs voix et le cri des soldats et les premiers tirs.

(...)

Tout se mêla sur les rives. Une vague d'assaillants reculait, décimée, et heurtant la nouvelle rangée qui partait à l'attaque, se joignit à elle, put avancer de quelques dizaines de mètres, tomba sous le feu de plus en plus précis des Allemands. Un autre pointillé humain se levait déjà et se lançait sur la pente glacée de la berge. Le crépitement de la fusillade devint continu, cadencé par des explosions, par les appels des commandants et les cris de blessés. De ce blessé-là surtout qui rampait toujours vers l'orchestre, poussant un râle déchirant et maculant la neige de son sang.

Le chant donnait à l'anarchie de toutes ces morts un rythme ample et grave qui semblait sonner au-delà du champ de bataille. Ils étaient peu nombreux sur leur scène de neige tassée, mais les soldats avaient l'impression que la puissance de tout le pays se soulevait derrière eux.

Ils reprenaient l'hymne pour la troisième fois quand Volski remarqua ces combattants qui venaient d'atteindre le haut de la rive opposée. Une rafale de mitrailleuse les faucha, mais leurs corps marquèrent la frontière la plus avancée de l'assaut. Il voyait tout, malgré la tension qu'exigeait le chant. Sur la glace de la rivière, des hommes s'agrippaient à l'affût d'un canon, à ses roues enlisées dans une 
congère. Leurs mouvements étaient à la fois fébriles et péniblement lents, comme ceux d'un coureur dans un mauvais songe (Makine, 2009 : 157-159). scène, rythmée par les voix des chanteurs et la tonalité des déflagrations et dont le procédé esthétique, mais aussi en quelque sorte ironique, nous rappelle le chapitre III du conte philosophique Candide ou l'optimisme (1759) de Voltaire qui dénonce les effets de la «boucherie héroïque ». Cette impression est d'autant plus forte que, plus tard, Makine critique le simulacre de guerre mis en scène par la propagande stalinienne par le biais duquel «les acteurs déguisés en soldats chantaient les exploits, l'héroïsme, la mère patrie » (Makine, 2009 : 207).

L'ensemble de ces procédés artistiques (photographiques, cinématographiques et musicaux $)^{3}$ permet non seulement de souligner le rôle central joué par le narrateurspectateur, mais aussi d'introduire deux idées essentielles se rapportant à ce dernier. Tout d'abord, le narrateur-spectateur de la guerre n'a pas vraiment conscience des horreurs qui se produisent autour de lui dans la mesure où elles font partie de sa vie quotidienne, ce qu'illustrent tout particulièrement les professions d'infirmière et de médecin de guerre qui effectuent leur travail sans trop se poser de questions, le nombre de blessés devenant en quelque sorte une abstraction liée à ce que nous pourrions qualifier de travail à la chaîne. C'est ainsi que Charlotte, dans le Testament français, dénonce un patient traumatisé par la guerre qui rouvre sa blessure chaque nuit à l'aide d'une éponge, sans penser à l'éventuel traumatisme vécu par le soldat. Quant au roman Requiem pour l'Est, il montre le mécanisme bien réglé des armes perfectionnées dont l'ajustement fait écho à l'existence « ordinaire » du narrateur. Les personnages, dans un premier temps, ont alors l'impression que les barbaries font partie de la normalité, à la manière de ces mines dont

les détonateurs (...), grâce à une astuce pneumatique, fonctionnaient seulement après plusieurs pressions, pour laisser le temps à une colonne de voitures d'entrer tout entière sur le champ miné. Une colonne de voitures ou une foule de femmes et d'enfants qui fuyaient leur village brûlé... (Makine, 2001 : 103).

ou encore de « ces drôles de balles avec le centre de gravité déplacé [qui] avaient l'atroce particularité de se promener dans le corps d'une façon imprévisible et de se loger aux endroits les plus difficiles à atteindre » (Makine, 2001: 37). Il n'y alors rien d'étonnant à ce que le ton des personnages nous paraisse plutôt détaché, voire neutre, alors qu'il devrait revêtir un caractère plus subjectif dans la mesure où il s'agit d'un narrateur externe.

L'absence de sentiments humains apparaît également dans la personnification des chars détruits par les bombardements qui incarnent le prolongement des souffrances subies par les soldats, des hommes broyés par la guerre. La description suivante se révèle particulièrement significative :

éventré par une roquette, il [le véhicule blindé] fumait encore en exhibant un mélange complexe d'appareils désarticulés, de métal tordu et de chairs humaines déchiquetées. La force de l'explosion avait rendu ce désordre étonnamment homogène, presque ordonné. Les fils électriques ressemblaient à des vaisseaux sanguins, le tableau de bord défoncé et éclaboussé de sang - au cerveau d'un être insolite, d'une bête de guerre futuriste. Et enfouie quelque part dans ce magma de mort, la radio, indemne, lançait ses appels chevrotants (Makine, 2001 : 27-28).

En dressant un parallèle entre les hommes et les machines - aussi bien des tanks que des trains (Makine, 1998: 138-139) -, Makine insiste sur la déshumanisation de l'individu, 
également symbolisée par les ruines des villes et des villages dont l'aspect calciné crée une vision « fantasmagorique » (Makine, 1998 : 137).

Mais, subitement, le narrateur-spectateur s'arrête sur une scène caractéristique dont l'image se grave dans sa mémoire, entraînant son "réveil » après un long endormissement et lui donnant à réfléchir sur le sens de la guerre, tant le choc est intense. Cette prise de conscience intervient aussi bien pendant la guerre qu'après le conflit. C'est alors que dans Confession d'un porte-drapeau déchu, quelques heures après avoir été pris au piège de ses propres tirs en série sur les Allemands - ces derniers ont créé un leurre -, le père de Kim comprend qu'il est devenu un «tueur " (Makine, 2009: $200)^{4}$, et ce depuis bien longtemps déjà : « Soudain, une idée toute simple et claire lui vint à l'esprit. Il pensa à toutes ces balles qu'il avait envoyées non pas dans des statues de sable mais dans des vivants. Jamais encore il n'avait pensé à cela...» (Makine, 1996: 36-37). Plus tard, c'est Kim lui-même, alors soldat, qui décide de sauver un enfant victime du lancement d'une grenade, désigné tout d'abord comme "un tas de chiffons qui remuait doucement» (Makine, 1996: 131), puis reprenant une forme plus humaine lorsque le narrateur le regarde plus attentivement : « le visage brûlé, les bras couverts de lambeaux de peau arrachée " (Makine, 1996: 131). Mais cet acte humain provoque aussitôt la colère de ses supérieurs habitués aux victimes, puis des années plus tard le désintérêt total des journalistes, plus préoccupés de leur stratégie commerciale que du destin de l'homme à proprement parler.

En ce qui concerne Charlotte, après une rencontre au cimetière avec la mère d'un soldat défunt, elle prend conscience de son acte de dénonciation vis-à-vis du jeune homme qui préférait s'automutiler pour ne pas devoir subir de nouveau les affres de la guerre. Elle en parle ainsi :

Il avait peur de revenir au front, et chaque nuit, il déchirait sa plaie avec une éponge. Je l'ai surpris, j'en ai parlé au médecin-chef. Nous avons mis à ce blessé un plâtre et quelque temps après, guéri, il repartait au front... Tu vois, à l'époque, tout cela me paraissait si clair, si juste. Et maintenant, je me sens un peu perdue. Oui, la vie est derrière moi, et soudain tout est à repenser. Ça te semblera peut-être stupide mais parfois je me pose cette question: 'Et si je l'ai envoyé à la mort, ce jeune soldat?' (Makine, 1998 : 255).

C'est à ce moment que les narrateurs-spectateurs décident de ne plus considérer la guerre et ses morts comme une habitude, sortant enfin de leur état léthargique pour passer à l'action et exiger que l'humain reprenne sa place. Leur prise d'initiative apparaît de deux manières. Premièrement, outre le fait d'abandonner des blessés à leur triste sort, le soldat refuse subitement de tuer l'ennemi qui n'est plus le symbole du camp adverse mais un être humain avant tout, ce dont témoigne Volski dans La Vie d'un homme inconnu à la fin d'une bataille :

Et soudain, surgie de la pénombre, cette silhouette chancelante: un tankiste allemand qui, sans doute abasourdi, s'en allait à l'aveugle au milieu des carapaces. Volski dégaina, visa... Mais ne tira pas. Le soldat était jeune et paraissait indifférent à ce qui pouvait lui arriver après l'horreur qu'il venait de vivre. Leurs regards se croisèrent et, malgré eux, ils se saluèrent. Volski rangea le pistolet, le tankiste disparut dans le crépuscule d'été (Makine, 2009 : 167-168).

Deuxièmement, le narrateur-spectateur se résout à dire la vérité consistant à s'insurger contre la cruauté de la guerre au travers de ces mêmes « détails » qui lui ont permis de s'insurger contre les injustices et les barbaries causées par les assassinats successifs. Afin d'engendrer également une révolte chez son lecteur, Makine introduit des faits 
dérangeants et des images choquantes que les textes de propagande préfèrent taire pour imposer une vérité supportable et surtout plus séduisante pour les masses.

D'une part, la description de ces images peut se révéler très brève lorsqu'il s'agit uniquement d'attirer l'attention du lecteur, formé aux récits de guerre "politiquement corrects ». Il s'agit, par exemple, d'un soldat victime d'un coup de sabre qui porte ses tripes dans ses bras, $d$ '« une jeune fille, une statue recouverte de givre (...) [qui] avait tenté de puiser de l'eau, s'était affalée, terrassée par l'épuisement» (Makine, 2009: 139), " un soldat aux yeux brûlés par une explosion [qui] cour[t] vers les chars, aveugle, guidé par le bruit des moteurs et par sa détresse, et roul[e] sous les chenilles en faisant sauter une grenade " (Makine, 2001: 188), d'" un jeune soldat [qui] apprenait à allumer une cigarette en la serrant entre les moignons de mains qui lui restaient» (Makine, 2001 : 194), d'un soldat Allemand dont « la main gauche, couverte de sang, était accrochée à la poignée de la mitrailleuse par un fil de fer qui remplaçait les ligaments déchirés et permettait le tir " (Makine, 2001: 224), d'un "jeune fantassin à la mâchoire inférieure arrachée et dont la langue pendait sur les pansements sales " (Makine, 1998:141) ou encore d'un blessé dont la plaie cachée sous un plâtre est remplie de vers blancs.

D'autre part, les passages énoncés peuvent faire l'objet d'un exposé plus long et approfondi lorsque Makine s'efforce de rompre avec les habitudes concernant les clichés utilisés pour évoquer la guerre, à savoir les scènes de combats héroïques, les corps déchiquetés par les explosions, les invalides de guerre et le sort des civils. Afin d'illustrer au mieux ce procédé, nous citerons trois scènes significatives qui «jouent » contre ces images banales et stéréotypées. Tout d'abord, concernant les batailles sanglantes, nous évoquerons celle des compagnies disciplinaires, composées de soldats déchus servant d'« appât » (Makine, 2001: 199), dont l'existence, au lendemain d'un mot malheureux émis sous un régime totalitaire, est devenue insignifiante et dont la société ignore le plus souvent l'histoire. Par conséquent, ces hommes, pourtant « exclus » de l'Armée, avancent à découvert et sans aide extérieure à la manière de pions sur un échiquier :

Deux cents soldats de sa compagnie avançaient droit sur les positions allemandes, sans aucun soutien d'artillerie, sans chars, sur une plaine nue, une mitraillette pour cinq hommes. Ils savaient que derrière eux, une section de barrage était prête à abattre celui qui aurait voulu reculer. On ne pouvait qu'avancer vers la mort ou reculer vers elle. L'unique choix (Makine, $2001: 198)$.

Puis Makine dénonce l'injustice dont sont victimes les invalides de guerre alors même qu'ils devraient être soutenus par le gouvernement dans la mesure où ils se sont sacrifiés pour leur patrie. Ainsi, dans Confession d'un porte-drapeau déchu, le père de Kim doit patienter des années pour obtenir la petite voiture lui permettant de se déplacer librement. En attendant, il doit se construire une petite carriole ou se laisser porter par son ami Iacha qui l'aide même à réaliser son rêve de vrai paysan, à savoir faucher les herbes des champs :

Iacha marchait à pas lents, rythmés, la tête et les épaules rejetées en arrière. Il nous tournait le dos. Dans ses bras croisés sur son ventre il portait mon père. Il ne l'avait jamais porté ainsi. Et mon père dans un geste large et libre maniait une faux. L'herbe frémissait et se couchait, dans un ample éventail argenté. Ils ne se disaient rien. Ils semblaient avoir trouvé leur cadence (Makine, 1996 : 157).

Mais la scène la plus révélatrice de la situation d'abandon subie par les mutilés de guerre, également méprisés par la société qui refuse d'ouvrir les yeux et de se confronter à la réalité, est vécue par Charlotte dans le roman Le Testament français qui rencontre des « samovars ", à savoir des «soldats sans bras ni jambes, [d]es troncs vivants" (Makine, 
1998: 144) dont la représentation physique rappelle celle de la théière russe traditionnelle :

Roulant dans leur caisse, dotée tantôt de petites roues avec des pneus en caoutchouc, tantôt de simples roulements à billes, ils abordaient les gens à la sortie, leur demandant de l'argent ou du tabac. Certains donnaient, d'autres accéléraient le pas, d'autres encore lâchaient un juron en ajoutant d'un ton moralisateur : "Déjà l'État vous nourrit... C'est honteux!». Les samovars étaient presque tous jeunes, quelques-uns visiblement ivres. Tous avaient des yeux perçants, un peu fous... (...) Charlotte s'arrêta, tira avec hâte un billet de sa sacoche et le donna à celui qui s'approcha le premier. Il ne put pas le prendre - sa main unique, la main gauche, n'avait plus de doigts. Il tassa le billet au fond de sa caisse, puis, soudain, il tangua sur son siège et tendant son moignon vers Charlotte, lui effleura la cheville. Et il leva sur elle son regard plein d'une démence amère...

Elle n'eut pas le temps de comprendre ce qui se produisit ensuite. Elle vit un autre mutilé, avec deux bras valides celui-ci, qui surgit à côté du premier et, brutalement, tira le billet froissé de la caisse du manchot. Charlotte poussa un « Ah », puis ouvrit de nouveau son sac. Mais le soldat qui venait de lui caresser le pied semblait résigné - en tournant le dos à son agresseur, il remontait déjà la petite ruelle très pentue dont le haut s'ouvrait sur le ciel... (...) Un bref cri rauque déchira la rumeur monotone qui planait au-dessus de la place.

Charlotte se retourna brusquement. La vision fut plus rapide qu'un éclair. Le manchot, dans sa caisse roulante, dévala la pente de la ruelle avec un crépitement assourdissant de roulements à billes. Son moignon repoussa à plusieurs reprises le sol en dirigeant cette descente folle. Et de sa bouche torturée par un horrible rictus, un couteau dardait, serré entre ses dents. Le mutilé qui venait de lui voler son argent eut juste le temps d'empoigner son bâton. La caisse du manchot percuta la sienne. Le sang gicla. Charlotte vit deux autres samovars se précipiter vers le manchot qui secouait la tête en lacérant le corps de son ennemi. D'autres couteaux brillèrent entre les dents. Des hurlements fusaient de toute part. Les caisses s'entrechoquaient. Les passants, médusés par cette bataille qui devenait générale, n'osaient pas intervenir. Un autre soldat descendait à toute vitesse la pente de la rue et, la lame entre les mâchoires, s'enfonçait dans le terrifiant entremêlement des corps mutilés... (Makine, 1998 : 257-259).

Dans ce passage, Makine insiste non seulement sur l'idée que le gouvernement délaisse les victimes de guerre, devenues un fardeau pour une communauté qui refuse de défendre des hommes infirmes leur rappelant la terrible réalité des assauts qu'elle préfère oublier, mais aussi sur le fait que les « samovars ", après avoir combattu pendant des années pour leur pays et cédé une grande partie de leur corps, doivent continuer de se battre pour survivre, cette fois-ci en temps de paix, et ce contre d'autres invalides aliénés par le désespoir et le rejet.

Enfin, dans Requiem pour l'Est, l'écrivain évoque les sévices infligés aux civils pendant des opérations punitives dont personne n'ignore l'existence mais que la communauté préfère taire de façon à ne pas avoir à l'affronter et, en quelque sorte, à la juger et à la condamner :

Ordonner aux captifs de creuser leur propre tombe et les enterrer vivants n'était pas rare durant cette guerre, il le savait. Ce qui le rendait perplexe c'était plutôt l'anarchie avec laquelle avaient agi les tueurs dans cette clairière. Certains des enterrés avaient le visage lacéré par un coup de sabre, l'un d'eux était décapité comme si son supplice ne suffisait pas. Nikolaï se dit alors que les enterrés s'étaient certainement mis à maudire leurs ennemis qui s'apprêtaient à partir et avaient ainsi provoqué ce massacre. D'ailleurs, ils avaient hurlé pour être tués, pour ne pas voir, le soir venu, les prudentes manœuvres des loups autour de leurs têtes sans défense (Makine, 2001 : 145-146). 
24

in l'ensemble des images utilisées par Makine permet de dénoncer les stéréotypes dépeints dans l'imaginaire de guerre habituelle. Les évocations de l'auteur semblent alors se rapprocher de celles d'artistes traumatisés par la guerre, notamment Otto Dix qui, en tant que soldat, a vécu la guerre non seulement de l'intérieur de par ses combats, mais aussi de l'extérieur lorsqu'il dessinait les soldats, les batailles et, plus tard, les invalides de guerre qu'il immortalisa dans plusieurs tableaux, tels Schützengraben (Tranchées) (1917), Die Skatspieler (Les Joueurs de skat) (1920), Streichholzhändler I (Le Marchand d'allumettes I) (1920), Der Krieg (La Guerre) (1924), Triptychon Der Krieg (1929-1932).

Comme Dix, Makine veut révéler l'horreur de la guerre à travers des images de laideur, destinées à provoquer le lecteur (ou le spectateur). Le recours au regard extérieur des personnages permet en effet de provoquer simultanément l'imagination du narrateur et du lecteur qui doivent se représenter l'inimaginable, se confronter à l'insoutenable et, surtout, y faire face sans se cacher une vérité qui dérange et n'est pas conforme au discours officiel ${ }^{5}$. Étrangement, le regard subjectif du spectateur devient donc objectif puisqu'il incarne le moyen de "vo[ir] le monde (...) avec un regard qui était incommensurablement plus grand que sa vue » (Makine, 2009: 201)) et de dénoncer «le jeu bureaucratique de[s] (...) diplomaties (...) [et] la démagogie de[s] (...) doctrines » (Makine, 2001:29).

En conséquence, à la question posée dans une émission littéraire télévisée dans le roman La Vie d'un homme inconnu: "Saura-t-on jamais dire la guerre dans un roman?», nous pouvons répondre que Makine a réussi à la décrire avec justesse et intégrité, délaissant cet "esthétisme qui devient obscène dans un livre sur la guerre " en choisissant de révéler des images chocs qui se veulent cependant différentes de celles exhibées par les médias qui cherchent seulement à faire monter l'audimat à des fins lucratives.

\section{BIBLIOGRAPHIE}

CLÉMENT, Murielle Lucie (2010). Le multilinguisme, la photographie, le cinéma et la musique dans son œuvre. Paris : L'Harmattan.

CLÉMENT, Murielle Lucie (2011). « [Entretien avec Andreï Makine] La littérature, science du salut » [on-line]. France : L'Obs (actualisé le 1/02/2011) [disponible le 25/04/2015]

URL: http://bibliobs.nouvelobs.com/romans/20110120.OBS6598/entretien-avec-andrei-makinela-litterature-science-du-salut.html.

LUBREZ, Geneviève (2005). « La réalité de la guerre dans Guerre et Paix et Requiem pour l'Est : un itinéraire de la guerre à la paix intérieure », in Margaret Parry, Marie Louise Scheidhauer, Edward Welch (éds.). Andreï Makine. Perspectives Russes. Paris : L'Harmattan, pp. 33-40.

MAKINE, Andreï (1996). Confession d'un porte-drapeau déchu. Paris : Gallimard.

MAKINE, Andreï (1998). Le Testament français. Paris : Gallimard.

MAKINE, Andreï (2001). Requiem pour l'Est. Paris : Gallimard.

Carnets, Deuxième série - 5 | 2015 
MAKINE, Andreï (2006). Cette France qu'on oublie d'aimer. Paris : Flammarion.

MAKINE, Andreï (2009). La Vie d'un homme inconnu. Paris : Éditions du Seuil.

NAZAROVA, Nina (2005). « Makine et Bounine : chanteurs de la Russie perdue », in Margaret Parry, Marie Louise Scheidhauer, Edward Welch (éds.). Andreï Makine. Perspectives Russes. Paris :

L'Harmattan, pp. 41-54.

NAZARova, Nina (2008). Andreï Makine, deux facettes de son œuvre. Paris : L'Harmattan.

VAREILLE, Arnaud (2009). « Du drame de devenir écrivain : La Confession d'un porte-drapeau

déchu d'Andreï Makine ", in Murielle Lucie Clément (éd.). Andreï Makine. Perspectives Russes.

Amsterdam-New York : Rodopi, pp. 37-53.

WANNER Adrian (2011). Out of Russia. Fictions of a New Translingual Diaspora. Evanston :

Northwestern University Press.

\section{NOTES}

1. Au vu des nombreuses références à la guerre en France, ces dernières nécessitent une étude à part entière.

2. De par ses peintures détaillées de la guerre, Makine peut même être comparé à un photojournaliste.

3. Pour plus de précisions à ce sujet, cf. l'ouvrage de Murielle Lucie Clément (Clément, 2010).

4. Cette expression est également employée par Volski dans le roman La Vie d'un Homme inconnu.

5. Il convient de noter ici que Makine utilise également des images poétiques liées à la nature ou à l'être humain, ce qui permet à l'écrivain de trancher encore plus fortement avec les terribles représentations de la guerre.

\section{RÉSUMÉS}

Dans ses œuvres, l'écrivain français d'origine russe Andreï Makine aborde à plusieurs reprises le thème de la guerre. C'est ainsi qu'il traverse les époques en évoquant aussi bien la guerre civile russe que les deux guerres mondiales. Alors même que l'auteur n'a pas vécu ces conflits, il les décrit de manière très réaliste, mettant en lumière des images d'horreur à la manière d'un chroniqueur de guerre objectif. Makine considère en effet ses romans comme la «fictionnalisation d'une idée », «basée sur une connaissance, sur un témoignage ». De ce fait, nous pouvons supposer que Makine a parlé avec plusieurs personnes ayant connu la guerre et/ou procédé à un travail de recherche approfondi effectué aux archives (de musées) dans le but de retranscrire les informations trouvées dans ses romans et de donner la parole à l'humain, s'opposant à la propagande contenue dans les mémoires et les manuels et préférant l'histoire individuelle à la grande Histoire.

In his works, the French writer of Russian origin Andreï Makine repeatedly deals with the topic of war. He crosses the boundaries of time to evoke the Russian civil war as well as the two world wars. Although the author did not live through these conflicts, he describes them in a very 
realistic way, drawing the most horrific pictures with the objectivity of a war columnist. Indeed, according to Makine novels are a "fictionalized idea", an idea "based on knowledge, on a testimony". As a result, we can assume that Makine spoke with several people who had experienced war and/or undertook a thorough search of the archives (of museums) with the purpose of retranscribing the information found in his novels and of giving voice to the human being. He therefore opposes the propaganda found in memoirs and textbooks, and favours individual history over great world History.

INDEX

Keywords : Makine (Andreï), war, horror, narrator-onlooker, awareness

Mots-clés : Makine (Andreï), guerre, horreur, narrateur-spectateur, prise de conscience

\section{AUTEUR}

\section{ALEXIA GASSIN}

Université Paris-Est Créteil

gas.alex@wanadoo.fr 